\title{
Topology of Three-Dimensional Active Nematic Turbulence Confined to Droplets
}

\author{
Simon Čopar, ${ }^{1, *}$ Jure Aplinc, ${ }^{1, *}$ Žiga Kos, ${ }^{1, *}$ Slobodan Žumer, ${ }^{1,2}$ and Miha Ravnik ${ }^{1,2}$ \\ ${ }^{1}$ Faculty of Mathematics and Physics, University of Ljubljana, Jadranska 19, 1000 Ljubljana, Slovenia \\ ${ }^{2}$ J. Stefan Institute, Jamova 39, 1000 Ljubljana, Slovenia
}

(Received 14 June 2019; revised manuscript received 2 August 2019; published 23 September 2019)

\begin{abstract}
Active nematics contain topological defects that (under sufficient activity) move, create, and annihilate in a chaotic quasi-steady state, called active turbulence. However, understanding active defects under confinement is an open challenge, especially in three dimensions. Here, we demonstrate the topology of three-dimensional active nematic turbulence under the spherical confinement, using numerical modeling. In such spherical droplets, we show the three-dimensional structure of the topological defects, which, because of closed confinement, emerge in the form of closed loops or surface-to-surface spanning line segments. In the turbulent regime, the defects are shown to be strongly spatially and time varying, with ongoing transformations between positive winding, negative winding, and twisted profiles, and with defect loops of zero and nonzero topological charge. The timeline of the active turbulence is characterized by four types of bulk topology-linked events - breakup, annihilation, coalescence, and crossover of the defectswhich we note could be used for the analysis of the active turbulence in different three-dimensional geometries. The turbulent regime is separated by a first-order structural transition from a low activity regime of a steady-state vortex structure and an offset single point defect. We also demonstrate coupling of surface and bulk topological defect dynamics by changing from strong perpendicular to in-plane surface alignment. More generally, this work aims to provide insight into three-dimensional active turbulence, distinctly from the perspective of the topology of the emergent three-dimensional topological defects.
\end{abstract}

DOI: 10.1103/PhysRevX.9.031051

Subject Areas: Soft Matter

\section{INTRODUCTION}

The ability of the material to employ externally or internally stored energy to spontaneously organize, flow, move, or change shape-i.e., be active-is found in a variety of materials [1]. A major class of active materials includes active nematics [2], which exhibit strong collective behavior and self-organization, emerging as local orientational order of material building blocks in material systems such as bacteria [3] and microtubule-kinesin mixtures [4,5]. Active nematic formalism could also be applied to describe other biological systems, such as cytoskeleton [6,7] and biofilm dynamics $[8,9]$. A recurring behavior in active nematics is the formation of topological defects, i.e., regions of broken orientational order, which are subject to topological-invariant conservation [10]. Because of strong localized deformation of the orientational order, some active nematic defects can perform as strong effective sources of material flow, dependent on the symmetry of the

\footnotetext{
*These authors contributed equally to this work.
}

Published by the American Physical Society under the terms of the Creative Commons Attribution 4.0 International license. Further distribution of this work must maintain attribution to the author(s) and the published article's title, journal citation, and DOI. defects $[11,12]$. A recurring property of topological defects in different active nematic systems is that at larger activities, they enter a regime of irregular and chaotic motion at low Reynolds numbers - called active turbulence. In the turbulent regime, generally, the topological defects are constantly created and annihilated $[3,4]$, which can be characterized by using statistical tools from classical turbulence $[13,14]$. The threshold between regular and irregular dynamics is strongly dependent on the confinement [15,16], higher-order force multipoles [17], and friction, which can even lead to stabilization of ordered defect phases in active nematics $[12,18]$.

Today, geometrical confinements are seen as one of the prime mechanisms for controlling the dynamics of active nematics [19-22], notably also in the context of possible energy harvesting [23]. Active nematics interact with their confinement through hydrodynamic boundary conditions, such as slip, no-slip, and friction, as well as through boundary conditions on the surface-imposed orientational ordering, also called surface anchoring. Active nematic droplets based on the mixture of microtubules and molecular motors were shown to periodically morph their shape [24]. Active nematic layers organized at the surface of spherical droplets are also explored from the perspective of topological defect trajectories and their mutual interactions [15,24-26]. When interfacing an ordered passive nematic fluid, active nematics can also exhibit directional streaming 
along the passive nematic director [27] and actuate the defects in the passive nematic host [28]. Fluid flow inside a droplet can produce net propulsion of the droplet, which itself acts as an individual active swimmer particle [29-31].

Realizing and controlling three-dimensional active nematic materials is an open emergent challenge in experiments [32-36] and numerical simulations [14,37-39], where one of the standing major complexities is how to characterise the complex 3D spatially and time varying flow and orientational fields that moreover have embedded topological defect regions [14]. Analogous systems exist that are dominated by topological defects in three dimensions, distinctly passive (nonactive) liquid crystal colloids $[40,41]$, and chiral ferromagnets $[42,43]$, where structures are controlled and characterized by using topological approaches. Indeed, because of the apolar nature of the nematic director, the nematic (passive or active) in three dimensions can form not only point defects (as in 2D) but also defect lines and loops, which can be characterized with different topological invariants, including winding number, topological charge, and self-linking number $[10,44]$. In passive nematics, coarsening structures during relaxation of a quenched state from isotropic to nematic also show a notable analogy in view of defect loop topology to 3D active turbulent defect profiles [45]. To generalize, the idea of this paper is to apply concepts of topology to characterize structural events in 3D active nematic turbulence.

In this paper, we explore the topological defect regimes of three-dimensional active nematics under the spherical confinement and no-slip surface, specifically focusing on the topology-affecting events in the active turbulence. We show that for moderate activities, the active nematic assumes an oriented state with an off-center shifted single point defect in the form of a small loop, and vortex flow with direction-set angular momentum. Upon increasing activity, we observe the onset of topological turbulence, characterized by a firstorder structural transition and hysteresis between the offset point defect and the turbulent regime, also showing the corresponding phase diagram as dependent on activity and droplet size. In the turbulent regime, we show that the active turbulent dynamics can be interpreted as a time series of topological defect affecting events: defect splitting, annihilation, merging, and crossovers. Depending on surface anchoring conditions, the defects are in the form of closed loops (perpendicular anchoring) or surface-to-surface lines that effectively wet the confining surface (in-plane anchoring), resulting in interesting surface-to-bulk conditioned turbulent dynamics. More generally, the results demonstrate dynamics of a strongly confined active nematic and point towards using concepts of topology for controlling the threedimensional active nematic turbulence.

\section{RESULTS}

The defect phenomena of the active nematic are explored in an elementary confinement of a droplet with fixed spherical shape by using mesoscopic numerical modeling of active nematodynamics $[2,11,28,46]$, which was shown to give good agreement with experiments on dense active nematic systems $[2,13,47]$. The approach relies on the dynamic coupling between the material flow and the mesoscopic order parameter tensor $Q_{i j}$ that covers the orientational ordering of active nematic. The activity is described by dipolarlike forcing via the active stress contribution. The confining spherical surface is set to impose strong perpendicular (homeotropic; in Figs. 1-3) or in-plane (degenerate planar; in Fig. 4) alignment of the active nematic at the surface, which, experimentally, would correspond to different surface functionalities [48]. Using strong anchoring and fixed spherical shape allows us to discern the effects of shape from the effects of topology that are otherwise inherently intertwined. Generally, such a fixed-shape regime corresponds to having materials with large surface tension or a background medium that is rigid enough to support the shape of the active nematic droplet (e.g., gel-like [49]). We use a no-slip boundary condition at the surfaces to simulate the host medium that resists flow. All distances are measured in units of the nematic correlation length $\xi_{N}$, time is measured in units of the intrinsic nematic timescale $\tau_{\mathrm{N}}$, and activity $\zeta$ is measured in units of $L / \xi_{N}^{2}$, where $L$ is the single elastic constant of the material. Equally, activity can also be described with the active length $\xi_{\zeta}=\sqrt{L / \zeta}$. Note that the nematic correlation length measures the effective size (thickness) of the defects and is given as a relative strength of the nematic elasticity vs variations in nematic order, which are-besides the activity and confinement-the key energetic mechanisms that affect the formation, structure, and dynamics of topological defects. For more on the approach, see the Appendix. Finally, note that this work focuses on the defect phenomena in a three-dimensional active nematic, i.e., the active nematic in the whole bulk of the spherical droplet, which is different from many current works that consider a thin layer of active nematic material, for example, at the surface of a droplet $[26,28]$.

\section{A. Active regimes in spherical droplet}

Depending on the activity of the material, two active regimes with distinct behavior of topological defects are found for the three-dimensional bulk active nematic in the confinement of a spherical droplet with perpendicular alignment: For low activity, structure with an offset stationary point defect is observed [Fig. 1(a) and Supplemental Video 1 [50] ], whereas for higher activities, a regime of threedimensional active turbulence with spatially varying and time-varying defect loops is observed [Fig. 1(c) and Supplemental Video 2 [50] ]. These two regimes are separated by a (hysteretic) structural transition [Fig. 1(b) and Supplemental Video 3 [50]]. In the limit of no activity (i.e., also no material flow), the considered spherical confinement would exhibit a radial nematic director structure with a single +1 radial hedgehog point defect at the center, as 
(a)

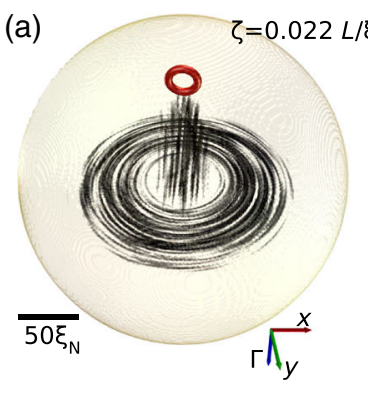

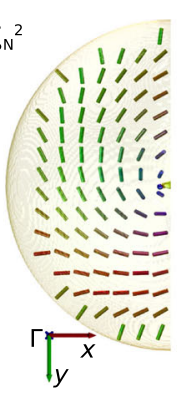

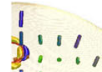

i:?!"

$11: . .2$

1 1....

11 i...

$\begin{array}{llll:}1 & 1 & 1 & \ldots \\ 1 & 1 & 0\end{array}$

$11: \cdots$

1: is

1 is

$\sqrt[y]{\Gamma}$

(b)
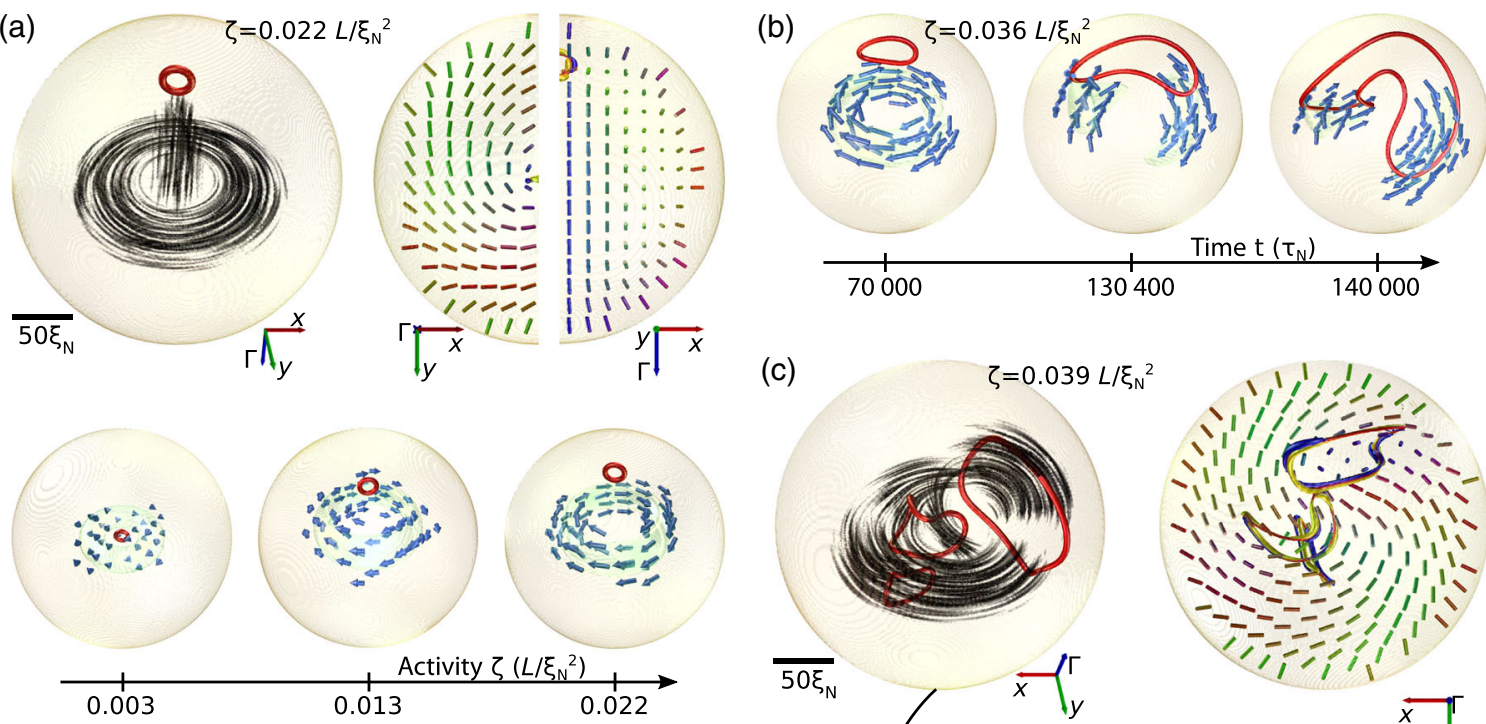

(d)

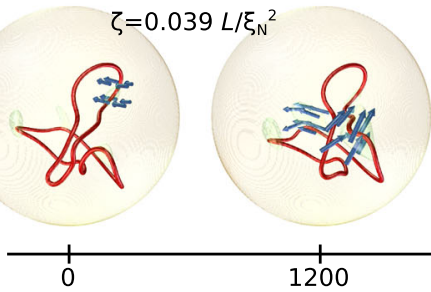

(c)
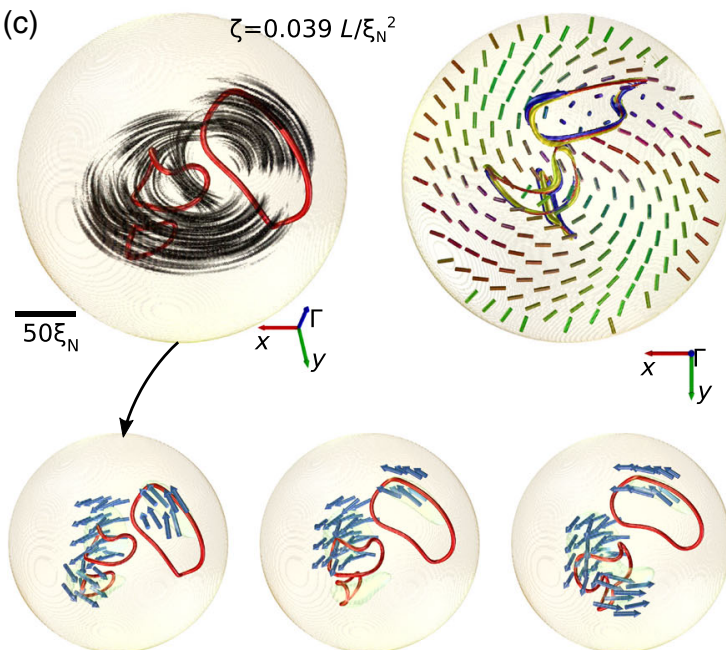

$\vec{x}$

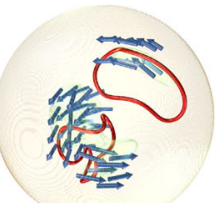

7000

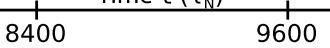

FIG. 1. Active nematic regimes in spherical droplet confinement with perpendicular surface alignment. Snapshots of dynamics for different activities, showing time evolution, velocity streamlines, and director field. (a) Active nematic regime at low activities with a single point defect (in red, in the form of a small ring) displaced from the droplet center and flow vortex (gray streamlines) with the angular-momentum vector $\boldsymbol{\Gamma}$. Note that the flow velocity component in the direction of $-\boldsymbol{\Gamma}$ is about 2.5 times smaller than the flow inplane component in the $x y$ plane. The right panel shows director profiles in the plane of the vortex and perpendicular to the vortex. Red corresponds to the director along the $x$ axis, green along the $y$ axis, and blue along $\boldsymbol{\Gamma}$. The bottom panel shows how, with increasing activity, the point defect (in red) moves further away from the droplet center and flow magnitude (blue arrows) increases. (b) Transition of the point-defect regime into the turbulent regime upon an increase of activity from $0.035 L / \xi_{\mathrm{N}}^{2}$ to $0.036 L / \xi_{\mathrm{N}}^{2}$; note the stretching and deformation of the defect loop and the disintegration of the regular velocity profile. (c) Turbulent active nematic regime. Defect loops are shown as isosurfaces of reduced degree of order (in red) and flow field with gray streamlines. The right panel shows the corresponding director in the given plane; the local variability of the defect cross section is illustrated with a splay-bend parameter (yellow and blue isosurfaces; see the Appendix). (d) Selected timeline of active turbulence in the active nematic droplet. The red isosurface of the scalar order parameter is drawn at $S=0.45$; blue arrows and bright green isosurfaces represent the velocity field above selected cutoff magnitudes (see the Appendix).

imposed by the confining surface conditions; indeed, such a profile is observed for activities below the activity $\zeta_{\text {onset }}=1.6 \times 10^{-3} L / \xi_{\mathrm{n}}^{2}$ (see also Fig. 2). Note that the hedgehog point defect appears in the form of a small ring of local winding number $+1 / 2$, with diameter of the order of nematic correlation length, and is topologically equivalent to a point [51]; the exact small-scale structure will, in practice, depend on the microscopic building blocks of the material. Therefore, in view of our work, loops with radius of the order of the nematic correlation length will be called point defects. Above the activity $\zeta_{\text {onset }}$, a selfsustained structure of a twisted director profile and a vortex flow appears, characterized by the orientation and magnitude of the angular-momentum vector [Fig. 1(a)]. Similar to (passive) cholesteric nematic droplets [52], the twisted director field pushes the point defect away from the droplet center. Since our system has no preferred chirality, the shift of the point defect can be both along and against the angular-momentum vector. Additionally, there is a velocity field component of roughly 2.5 times smaller magnitude than the circular vortex flow, which goes along the center of the droplet towards the point defect and then near the surface back to the opposite end of the spherical droplet confinement. For increasing activity, the magnitude of the vortex flow increases, and the defect shifts more and more toward the surface of the droplet; as a critical activity is reached, the small defect ring opens into a larger loop [see Fig. 1(b)]. At this value of the activity, the activity-induced flow can overcome the elastic barrier of the nematic field to transform the point defect into a topologically equivalent 

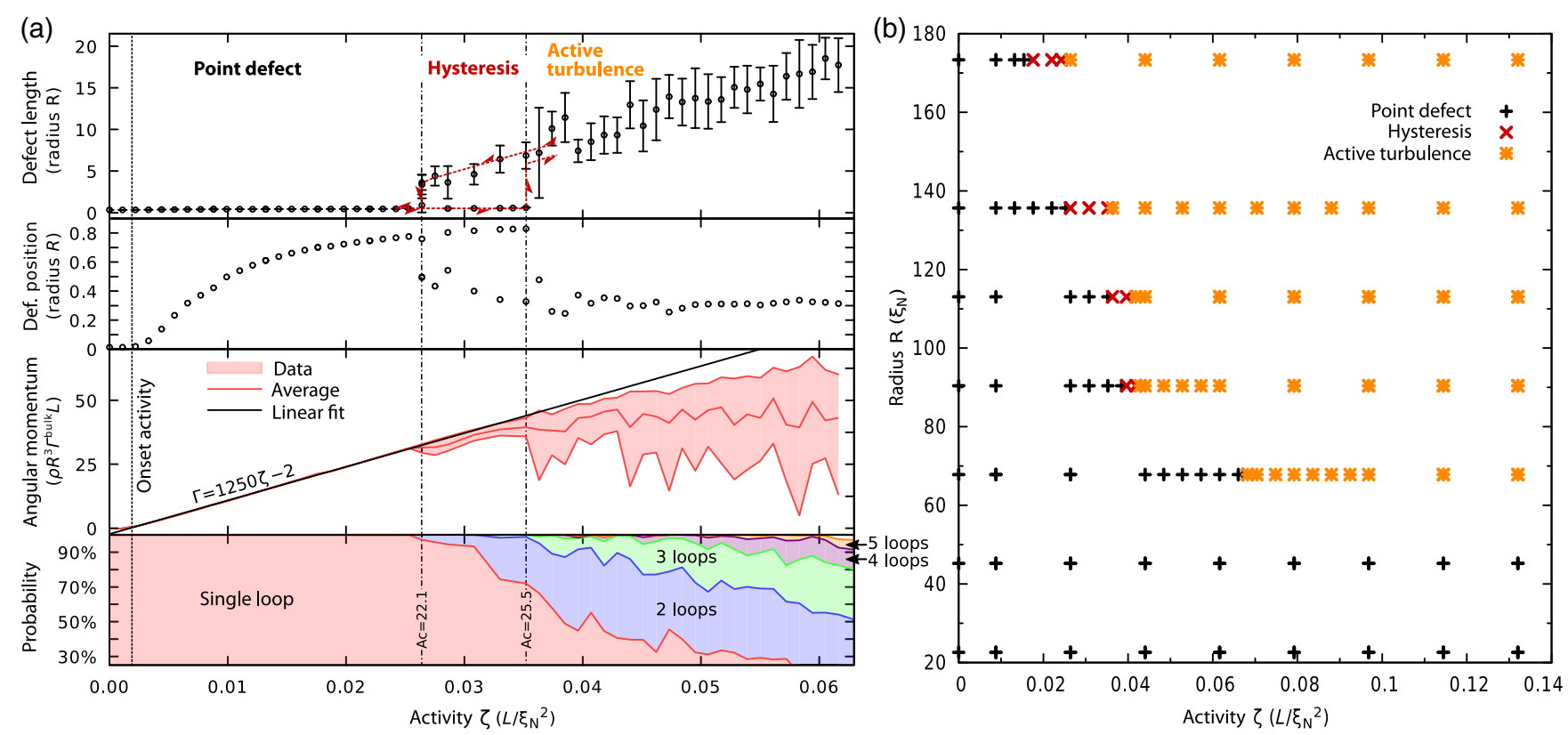

FIG. 2. Analysis of active nematic regimes in the confinement of a spherical droplet. (a) Multiple material characteristics of the threedimensional active nematic in spherical droplet confinement of radius $R=136 \xi_{\mathrm{N}}$, with perpendicular surface alignment dependent on the activity. The calculations are performed for increasing or decreasing activity, clearly showing a pronounced hysteresis. At each activity change, previous structure is taken as the initial condition, and simulation is run for $23000 \tau_{\mathrm{N}}$ for each data point. The top panel shows the total length of defect loop cores at a given activity, with the bars indicating the standard deviation of the natural oscillation of the defect length over time. The second panel in (a) shows the average radial distance of the defect core regions from the droplet center. The third panel gives the total angular momentum set by the flow and its standard deviation in time. The bottom panel gives the probability for finding the given number of defect loops within the droplet. Note three active regimes-point defect, hysteretic regime, and developed active turbulence — each with a different response of the active material characteristics. The linear fit of the angularmomentum dependence on the activity, in the point-defect regime, is used to set the onset activity below which the vortex flow profile has negligible magnitude and the point defect remains at the droplet center. Graphs of angular momentum and probability are shown only for hysteresis behavior at decreasing activity. The hysteretic region is delimited by activity numbers Ac $\approx 22.1$ and Ac $\approx 25.5$, for the considered droplet size. (b) Phase diagram of active regimes in spherical droplets with perpendicular surface alignment, as given by droplet radius $R$ and activity $\zeta$.

large defect loop. This process involves contributions of defect core line tension, nematic elasticity, advection, backflow, shear active flow, as well as coupling to confining geometry and actual local and global defect topology. Above the critical activity, the active nematic transitions into a chaotic irregular behavior-i.e., the active turbulence regime. There can be strong material flow due to large elastic deformation around the core of the loop, and overall, strong dynamic deformations of the loop are observed, including topology-modifying events, such as the breakup of a loop into multiple loops, as shown in Figs. 1(c) and 1(d).

Performing a more quantitative study of the active nematic regimes in the spherical droplet confinement, several distinct material characteristics are analyzed (as shown in Fig. 2) that can assess the three-dimensional nematic orientational ordering and material flow, as well as the topology of the defects. For each simulation, we changed the activity between two neighboring data points in Fig. 2(a); we let the system evolve for $4500 \tau_{\mathrm{N}}$ to reach a dynamic steady state and then over a time interval of
$18500 \tau_{\mathrm{N}}$ collected data on total defect length, (average) defect position, total angular momentum, and probability for observing $N$ number of defect loops [Fig. 2(a)]. Note that, in the analysis, we employ direct 3D tracking of defect line cores based on variations in the nematic degree of order, as further explained in the Appendix. The defect length inside the droplets quantifies that, at low activities, there is a regime of a single point defect, but upon increasing activity, a structural transition occurs in the active turbulent regime, in which the single defect loop or multiple loops dynamically transform and vary in time, with the total defect line length changing in time for the order of multiple $10 \%$ from the average value. The position of the center of the point defect changes with increasing activity and shifts from the droplet center towards the surface of the droplet. As the active nematic transitions to the turbulent regime, the active defect loops explore and transverse the available volume irrespective of actual activity. The average radial position of defect segments from the droplet center settles in the turbulent regime to approximately $0.3 R$, as at high activities the defect segments become more uniformly 
(a)
O Split
O Annihilation
- Merge
Crossover
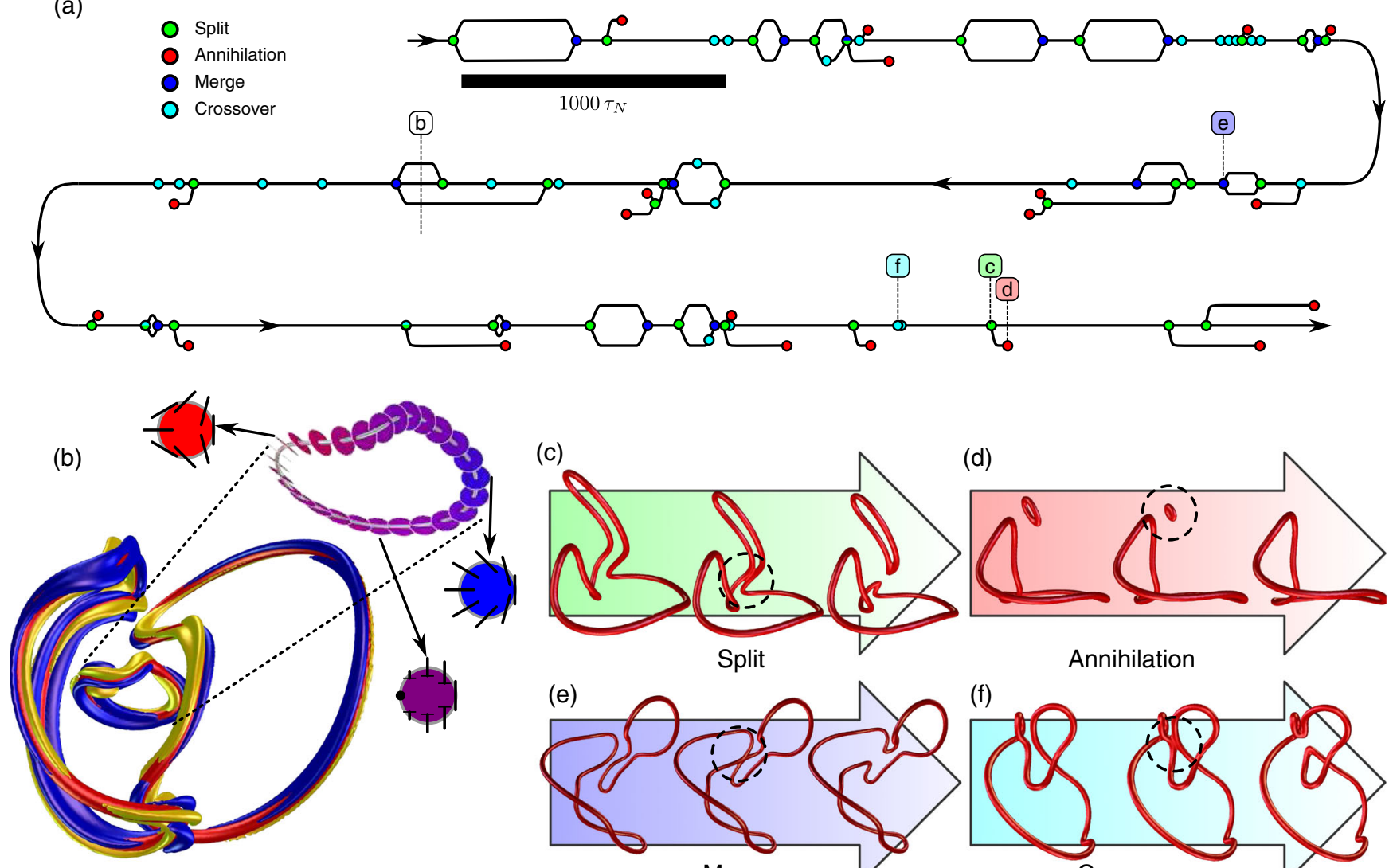

(c)

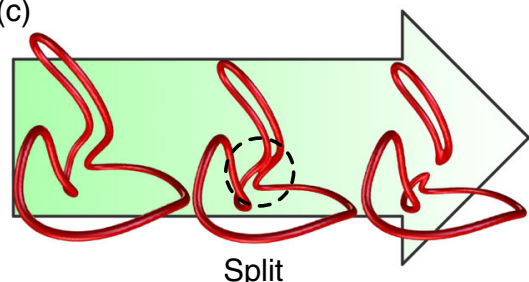

(e)

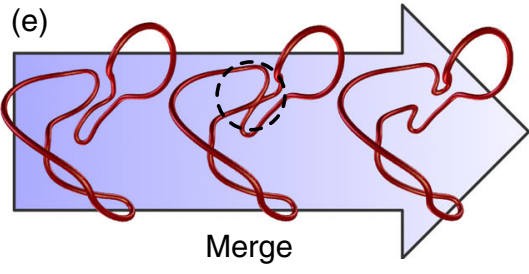

(d)

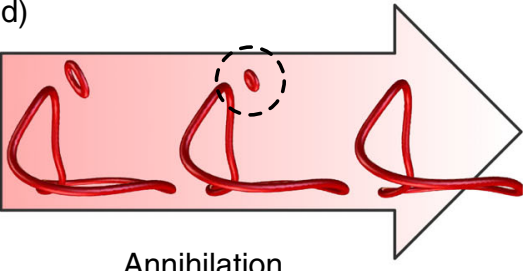

Annihilation

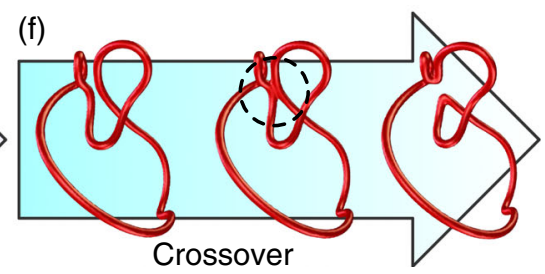

FIG. 3. Topological events in turbulent regime. (a) Graphically depicted topological flow diagram for the active nematic defect loops in a selected time interval. Note that the diagram time axis is to scale (black line) and is given in units of the intrinsic nematic timescale $\tau_{\mathrm{N}}$. At all times, one of the loops carries topological charge +1 , and the others have zero charge. (b) A snapshot of active turbulence with three loops. The color coding of the disks for the selected defect loop shows the type of local profile of the defect line, where blue refers to $+1 / 2$, red to $-1 / 2$ winding number, and purple to the local twist defect line profile. The variation of the defect line cross section is also illustrated more qualitatively with the splay-bend parameter (yellow and blue isosurfaces). (c)-(f) Time sequences of selected loop topology-changing events: splitting, annihilation, merging, and crossover. Panel (c) shows splitting of a neutral loop (upper) from a loop with a +1 topological charge (lower). The neutral loop soon annihilates in panel (d). The droplet radius is $R=136 \xi_{N}$, and the activity is $\zeta=0.04 L / \xi_{\mathrm{N}}^{2}$.

distributed. The average defect position also shows hysteretic behavior. The average defect core position from the droplet center measures well the point-defect position in the low activity state, but in the turbulent regime, it is more meaningful to observe the probability density of defect line segments per unit volume, with respect to relative distance from the center of the droplet, as shown in Fig. 1 in Ref. [50]. The angular momentum grows within the point-defect regime continuously with activity, according to a linear dependence $\left(\zeta-\zeta_{\text {onset }}\right)$. Note that $\zeta_{\text {onset }}$ is the activity for the onset of vortex flow, which we determine numerically by a linear fit of the angular-momentum dependence on activity. Note that such emergence of active flows in confined and defect systems is known to depend on the symmetry and profile of the equilibrium (no-activity) structure [53-55]. When the active nematic passes to the active turbulence regime, the average magnitude of the angular momentum gradually saturates with activity, which again signifies the breakup of the regular defect dynamics. At this transition between the point-defect and active turbulence regimes, the process of defect loop breakup (i.e., creation of new loops) and annihilation of emerging loops also begins, resulting in a growing fraction of time when there is more than one loop present in the spherical droplet.

Activity is the main control parameter for the transition between the point-defect regime and the active turbulence; however, this transition could also be driven, for example, by changing the active nematic elastic constant or the size of the confining cavity. Figure 2(b) presents a phase diagram of active regimes with respect to activity and droplet size, showing that larger droplets transition into the active turbulent regime at lower activities, whereas smaller droplets require larger activities to undergo structural transition from regular to irregular dynamics. Indeed, such behavior can be well understood by considering the dimensionless activity number $\mathrm{Ac}=\sqrt{\zeta R^{2} / L}$, which 


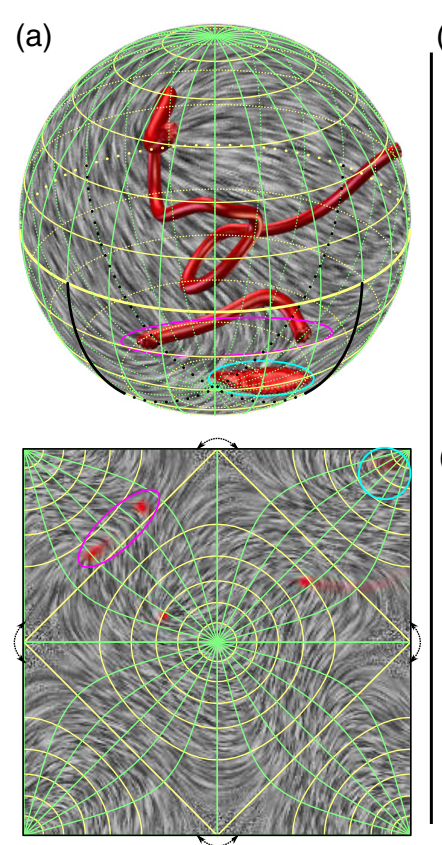

(d)

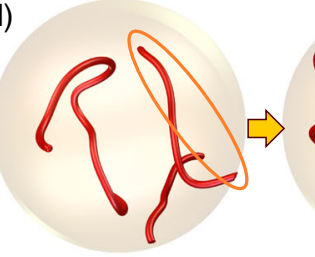

(b)
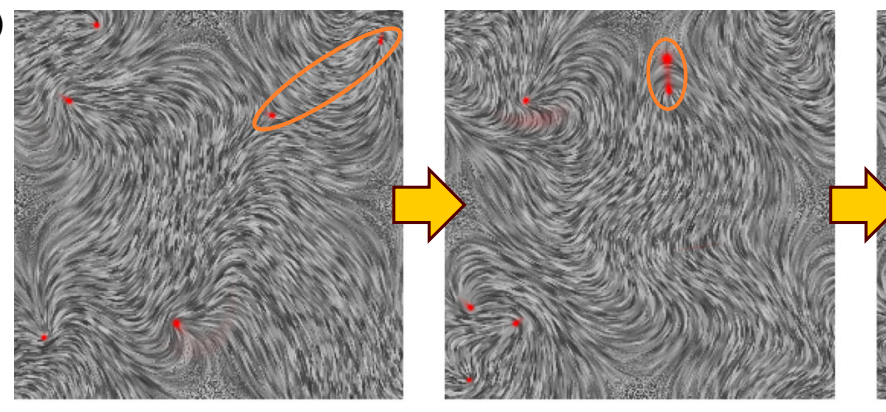

(c)

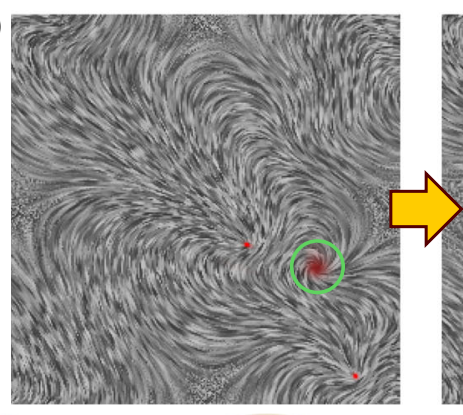

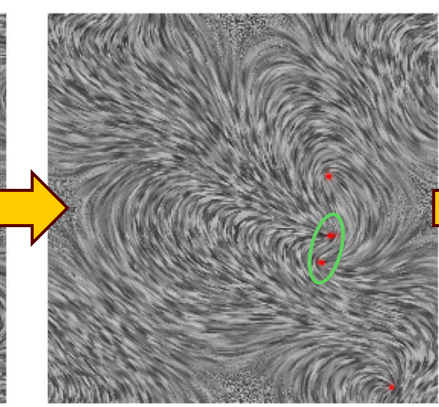

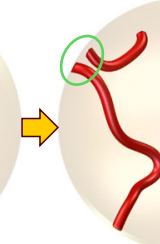

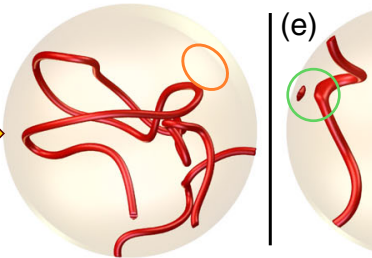
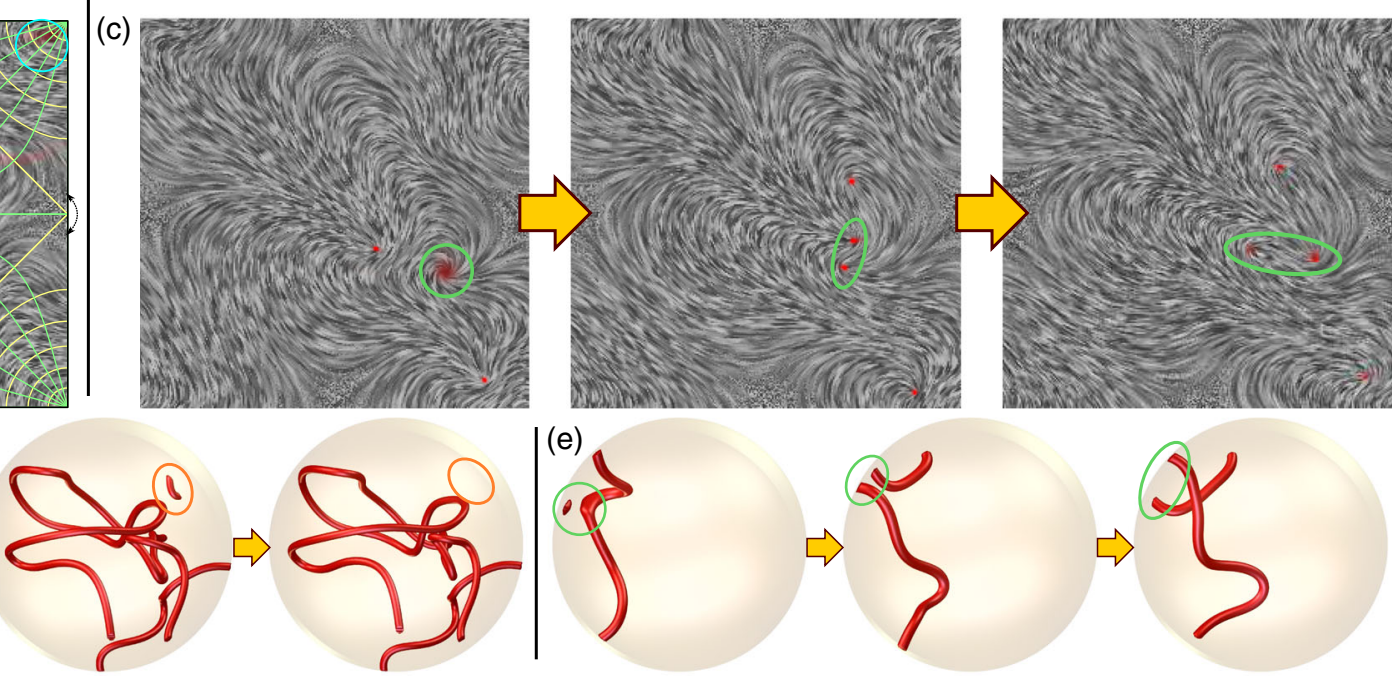

FIG. 4. Surface and bulk active nematic topological defect transitions in spherical droplets with planar degenerate surface alignment. (a) Snapshot of an active nematic droplet showing two bulk defect lines, which terminate at the surface (ends of one are encircled with magenta), and a boojum defect (encircled in cyan). The in-plane director field at the spherical droplet surface is represented by grayscale streaks. The bottom panel shows the Peirce quincuncial projection map of the surface director and defects from the entire sphere onto a square; the sphere can be reproduced by folding four corners of the square back together into one point, where the yellow square represents the equator of the sphere. Note the pointed out defects encircled in magenta and cyan, as shown in the sphere and the projection square. (b) Annihilation sequence of a selected $\pm 1 / 2$ defect pair at the surface of the active nematic droplet shown using the Peirce projection map. During the sequence, a surface profile with five $+1 / 2$ defects and one $-1 / 2$ defect is transformed into a four $+1 / 2$ defect profile, conserving the +2 surface topological charge set by the Euler characteristic of the sphere. In the bulk of the droplet, the sequence corresponds to a line segment being annihilated on the surface, as shown in (d). The annihilating surface defect pair is encircled in orange. (c) At the droplet surface, +1 surface boojum breaks into two $+1 / 2$ defect profiles (encircled in green). In the bulk, this surface process corresponds to a defect line segment interacting with the surface boojum, resulting in two bulk defect lines, as shown in (e). (d) Full view of the changes in the bulk defect structure (in red, drawn as isosurfaces of $S=0.51$ ) that corresponds to the defect annihilation shown in (b). (e) Full view of the changes in the bulk defect structure (in red, drawn as isosurfaces of $S=0.523$ ) that corresponds to the breaking of a boojum shown in (c).

gives the ratio between the confinement size $R$ and active length $\xi_{\zeta}$. In Fig. 2(b), the transition from the point defect to active turbulence is observed roughly at Ac $\sim 20$.

The structural transition between the point-defect structure and the active turbulence exhibits a clear hysteresis, which is a signature of the discontinuous (first-order) transition between the two structures [Fig. 2(a)]. The ordered phase with the offset point defect can be "overactivated" (i.e., with activity above the transition value), which we simulate by gradually increasing activity in small steps from the low activity state until the turbulence appears, assuring, for each step in the activity change, that the system reaches the dynamic steady state. Alternatively, the turbulent state can be "underactivated" (i.e., with activity below the transition value) before the turbulent defect loop collapses into a point defect, which we simulate by gradually decreasing activity in small steps. The turbulent state can only appear when the flow is strong enough to overcome the elastic tension force of the defect line. Once the defect line is extended, it can remain extended even at lower activities. In the turbulent regime, the system behaves chaotically, where changes in the shape of defects can lead to a very different future evolution of the system. The system has no static equilibrium, but it reaches a dynamical steady state in which macroscopic variablessuch as the defect length, angular momentum, and average 
number of loops-settle to fluctuate around an average value.

\section{B. Topology of defect loops in active turbulence}

Topology is a natural tool for studying topological defects that goes beyond exact detailed material characteristics and focuses on the overall structure; we use topological analysis for the characterization of the active turbulent regime under the confinement of the active nematic into spherical droplets. We focus on topological properties of the actual defect loops-their number, how they are connected to each other, and their topological charge-as well as on the local variation of the nematic director surrounding the defect loops, i.e., considering defect loops not only as lines but also as objects with internal structure. Figure 3 shows the dynamics of active nematic turbulence in the spherical droplet, analyzed from the perspective of the topology of three-dimensional active defect loops. In Fig. 3(a), a time series of defect topologyaffecting events is presented-importantly, with the time axis in scale-for a selected time interval of the active turbulence in an active nematic droplet $\left(R=136 \xi_{N}\right.$ and $\left.\zeta=0.04 L / \xi_{\mathrm{N}}^{2}\right)$. Four different topological events are observed to occur stochastically in time, with the average event rate set by (and increasing with) the activity. New loops of zero topological charge are observed to detach (pinch off) from a preexisting loop (the existence of at least one loop is topologically enforced by the droplet's perpendicular surface conditions); extra loops annihilate or merge back into the loop with nonzero charge, which by itself cannot disappear. Crossovers of the defect loop with itself can rewire the loop, reversing the path orientation of one part relative to the other. More generally, the visualized map reflects the conservation laws that would formally be described with a Morse theory, where topological events are critical points (saddles, endpoints) of the manifold comprised of the defect loci over time (the world sheet) [56]. Examples of loop splitting, annihilation of a single loop, merger of two loops, and a crossover (i.e., self-intersection) are shown in snapshots in Figs. 3(c)-3(f) and in more detail in Fig. 2 in Ref. [50].

The director cross section of defect loops in the regime of active turbulence varies along the defect loop in a complex way and also changes in time, as shown in Fig. 3(b) and in Fig. 3 in Ref. [50]. The defect loops in the threedimensional active turbulent regime exhibit sections of local director symmetries of $+1 / 2$ and $-1 / 2$ winding number and twisted profile, which has a profound effect also on the local dynamics and local active flow. It is well known from $2 \mathrm{D}$ active nematic systems that $+1 / 2$ defect profiles are generally strong generators of flow and that they strongly propel this type of (in 2D) point defects, whereas $-1 / 2$ defect profiles do not generate flow and are less motile [11]. Indeed, it is this variability of the director field that we now observe in the local cross sections of the three-dimensional defect loops (see Fig. 3 in Ref. [50]), which then affects the dynamics of not only itself but all defect loops, through both nematic elasticity and hydrodynamics. The role of local cross sections is known to affect the dynamics of reconnection events between defect line segments $[57,58]$. In our system, immediately before and after topological reconnection events, the director orientations of both defect line segments generally lie in the same plane and rewire in a way that makes the director between the defect lines uniform (without introducing topological solitons). For example, two defect line segments with a locally twisted profile may reconnect into another pair of twisted profiles, a pair of $+1 / 2$ and $-1 / 2$ local profiles, or other intermediate profiles with a net neutral winding number (see Fig. 2 in Ref. [50]). Finally, the fact that the defect lines in spherical droplet confinement with perpendicular conditions are closed into loops imposes that this local director profile $(+1 / 2,-1 / 2$, or twisted) must come back to the same orientation when encircling the loop, which encodes the total topological charge carried by the loop [44].

At the level of the topology of the loop as a whole with the surrounding director, we observe that one of the loops always carries a +1 topological charge - set by the +1 topological charge imposed by the perpendicular (homeotropic) surfaces-whereas the rest are topologically neutral (i.e., charge zero) and can annihilate without contacting another loop [see Fig. 3(d)] or, alternatively, merge back with another loop. From a purely topological perspective, pairs of oppositely charged loops could also be formed (not observed in our simulations), especially in larger systems and with larger activities, while still preserving the net charge conservation set by confinement. However, the formation of oppositely charged pairs is - at least in the considered material regime - energetically even more unfavorable as than the formation of zero-charged defect loops. Finally, a possible further approach to classify the active defect loops topologically could involve the quaternionic approach [44] or construction of Pontryagin-Thom surfaces [59].

\section{Surface-contacting topological defects in active turbulence of spherical droplets with in-plane alignment}

Active turbulence in active droplets of fixed spherical geometry with strong surface degenerate in-plane alignment (i.e., planar degenerate anchoring) is explored, showing a system of interesting surface-to-bulk coupled defect dynamics. Under degenerate planar anchoring, the defect lines within the bulk are not necessarily closed into loops but can also terminate at the surface, as shown in Fig. 4 and Supplemental Video 4 [50]. Indeed, in addition to closed defect loops observed for droplets with perpendicular surface alignment, defect lines with surface-to-surface spanning ends and surface boojum defects are observed, resulting in the turbulent defect dynamics; in the bulk, this dynamics is 
generally similar to the dynamics in homeotropic droplets, but it is additionally coupled to the topological events at the droplet surface. Figure 4 shows two such surface events using the Peirce quincuncial projection of the surface director and defects from the sphere onto a square: In Figs. 4(b) and 4(d), a pair of $\pm 1 / 2$ defects within the surface director field is annihilated, and in Figs. 4(c) and 4(e), a surface boojum is split into a pair of $+1 / 2$ defects. During the pair surface annihilation event, a structure with four $+1 / 2$ defects and $a \pm 1 / 2$ defect pair is transformed into a structure with only four $+1 / 2$ defects. In the bulk, such an event corresponds to either (i) the annihilation of a defect surface-to-surface line segment on the surface of the droplet [which was the actual case in the presented simulation, as shown in Fig. 4(d)] or (ii) a merger of two ends of defect line segments into one segment and the following detachment of the defect segment from the surface. In Fig. 4(c), a +1 surface boojum interacts (and merges) with a section of the bulk defect, which results in splitting of the boojum into two $+1 / 2$ defects, transforming the surface director profile with two $+1 / 2$ defects and the +1 boojum into a surface field with four $+1 / 2$ defects. In the bulk of the droplet, the defect line that merged with the boojum splits into two parts, each of them terminating at one of the newly created $+1 / 2$ defects at the surface, as shown in Fig. 4(e). Additionally, not shown here, we observe creation of surface defect pairs when an existing bulk defect loop segment is pushed from the bulk to the surface, where it is split into two ends (seen at the surface as a pair of $\pm 1 / 2$ defects) or, alternatively, a reconfiguration of the surface charges by merging of combinations of $-1 / 2$ defects and +1 boojums.

Topologically, the observed active turbulent defect dynamics in droplets with in-plane alignment is determined by the director on the surface where the total winding number (2D topological charge) must be equal to the Euler characteristic of the confining surface $\chi$. Specifically, in our droplets, the net surface topological surface charge must equal $\chi=2$, which in the limit of zero activity, is realized by a pair of +1 boojums at the opposite poles of the droplet. However, for nonzero activity, as shown if Fig. 4, the surface boojums are only rarely observed, and the surface charge is distributed between four $+1 / 2$ defects and additional pairs of $\pm 1 / 2$ defects, which are actually ends of the bulk $+1 / 2$ or $-1 / 2$ defect lines touching the droplet surface. More generally, the topological dynamics of the defect lines (and loops) in the 3D bulk of the active nematic induces a lower-dimensional active defect dynamics at the confining surfaces.

The turbulent defect dynamics at the surface of the droplet shares similarities with the 2D active nematic turbulence (e.g., in films or shells); however, it is different because it is actually coupled to the active flow and defect reconfiguration of the entire bulk. The no-slip boundary condition for the material flow, used in this work, effectively prevents surface defects from being driven by the activity directly on the droplet surface, but it is the active dynamics of bulk defects that cause apparent active motion, creation, and annihilation of surface defects in the turbulent regime as provided via effective elastic line tension and splitting of bulk defect lines at the surface. Nevertheless, in different realizations, we very much envisage that the velocity boundary condition could be different-from no slip, to partial slip or (full) slip—in which case, one would expect similar defect dynamics but now also propelled through the surface. Alternatively, the defect dynamics might also be affected by different surface and bulk rotational viscosities, effectively causing the defects in the bulk and at the surface to move at different timescales. Finally, such combined actively driven surface and bulk dynamics might lead to the emergence of effectively coupled or decoupled dynamics of defects at the surface and in the bulk.

\section{DISCUSSION}

Today, confinement is seen as one of the major routes for controlling active matter-including active nematics — with multiple geometries explored [19-21,24]. From the perspective of this work, we should emphasize that we purposely chose the regime where the droplet radius $R$ is generally comparable to but larger than the active length $\xi_{\zeta}=\sqrt{L / \zeta}$, i.e., $R / \xi_{\zeta} \lesssim 35$, and the surface interactions (surface anchoring) are strong, such that the confinement has a profound role. The main effect of confinement with the strong homeotropic boundary condition is that it enforces the existence of at least one bulk topological defect (loop) by imposing non-net-zero topological charge in the bulk of the droplet, and similarly, strong planar anchoring imposes nonzero surface topological charge, thus enforcing the existence of surface defects. This confinement-imposed existence of defects in the limit of low activity has a notable effect on the onset of active turbulence as it circumvents the need for spontaneous creation of defect pairs. Instead of a spontaneous defect creation, existing defects get transformed-e.g., existing defects split into defect pairs. As a result of this lowerenergy barrier mechanism, in this work, we do not observe events where new defects would emerge from an instability of the homogeneous nematic field or would involve the spontaneous appearance of a single loop with zero topological charge; defect loops are instead created by a breakup from an existing defect loop. In a similar manner, one could envisage that some surface anchoring profiles or structures with sharp edges, which would cause strong local distortions of nematics, could perform as nucleating regions for the emergence of the active nematic defects. Finally, the topology and geometry of the confining surfaces thus can play an important part in generating distinct active regimes.

The major body of current work in active nematics is for two-dimensional or quasi-two-dimensional regimes and geometries [3,4]. From the perspective of topological defects, the key difference between $2 \mathrm{D}$ and $3 \mathrm{D}$ active 
nematics is that the defects change from defect points in plane to defect lines and loops in three-dimensional space, which introduces a notable increase in the complexity of tracking and monitoring approaches for the defects. Topologically, the key difference is that, in 2D nematic fields, only $+1 / 2$ and $-1 / 2$ defects are possible, whereas in 3D nematics, the defects can be in the from of points, loops, or walls, and even lines if they can terminate appropriately, like on surfaces. Also, solitonic solutions are starting to be seen in objects like active skyrmions [60]. These three-dimensional nematic defect structures can be characterized with different topological invariants (in 2D nematics, there is only 2D topological charge) that account for the topology of the defects as a whole, such as the 3D topological charge, linking, and self-linking numbers. Defects can also be characterized locally through their cross section, for example, with the winding number. At the level of orientational order fields, active nematics are topologically equivalent to the passive (i.e., not active) nematics, in which 3D defect structures as complex as knots and links were demonstrated [40,41]. It is an interesting question whether similarly complex three-dimensional topological defect structures could also emerge in active nematics and, moreover, if they could have some role in real living matter.

In conclusion, using numerical modeling, we demonstrate the topology of three-dimensional active turbulence in droplets with fixed spherical shape of active nematics, considering both droplets with perpendicular and in-plane imposed surface orientation. Structural transition with developed hysteresis between the bulk regular offset point-defect regime and the irregular regime of active turbulence is shown for homeotropic droplets. Defect length, defect position, angular momentum, and number of defect loops are used as bulk material characteristics that can account for the coupled material flow, orientation, and topological variability of the three-dimensional active nematic in the turbulent and nonturbulent regimes. The bulk active defect lines and loops are shown to exhibit local profiles with spatially and in-time varying cross sections, with winding numbers of $+1 / 2,-1 / 2$, and twisted profile. Individually, defect loops are generally topological-charge neutral, except for compensating for the charge imposed by the confining surface. The topological flow diagram is shown as a strong tool for topological characterization of active turbulence, identifying a distinct set of topology-affecting or changing events - defect splitting, annihilation, merging, and crossovers-with their conservation laws drawing on an analogy with Morse theory. For droplets with degenerate in-plane surface alignment, we show that active defects can touch (wet) the surface, creating active defect line segments that span between different surface regions, topologically coupling the surface and bulk defect dynamics. More generally, the aim of this work is to approach - in full three dimensions - the complex temporal and spatial variability of active matter using a topological toolbox and, moreover, to explain the observed continual dynamics in view of distinct transitions between selected well-characterized topological structures.

\section{ACKNOWLEDGMENTS}

The authors acknowledge discussions on 3D active defect lines with D. Beller. The work was supported by the Slovenian Research Agency (ARRS) under Contracts No. P1-0099, No. J1-9149, No. N1-0124, and No. L18135, and COST action EUTOPIA (CA17139). M. R. also acknowledges support under EPSRC Grant No. EP/ R014604/1 at Isaac Newton Institute, University of Cambridge ("The mathematical design of new materials" program).

S. Č., J. A., and Ž. K. contributed equally to the work. J.A. and Ž.K. performed numerical simulations and analyzed the results. S. Č. performed geometric and topological analysis. $\check{Z}$. K. developed numerical code. M. R. and S. Ž. supervised the work. All authors contributed to writing of the manuscript. M. R. initiated and led the research.

\section{APPENDIX: METHODS}

\section{Modeling of active nematics}

We apply a mesoscopic continuum description of (dense) active nematic, which is based on coupled dynamic equations for orientational order and the material flow field $[2,28,46]$. Roughly speaking, this approach is based on the material flow generation via the active forces caused by the distortions in the orientational ordering of the active nematic, and the back-coupled response of the active nematic orientation to the generated flow. The nematic ordering is described by a traceless tensor order parameter $Q_{i j}$ whose largest eigenvalue is the degree of order $S$ (also called a scalar order parameter), and the corresponding eigenvector gives the main ordering axis, called the director n. The dynamics of the Q-tensor is given by the adapted Beris-Edwards equation [61]

$$
\left(\partial_{t}+u_{k} \partial_{k}\right) Q_{i j}-S_{i j}=\Gamma^{\text {bulk }} H_{i j},
$$

where $\partial_{t}$ is a derivative over time $t, \partial_{k}$ is a derivative over the $k$ th spatial coordinate, $\boldsymbol{u}$ is the fluid velocity, and $\Gamma^{\text {bulk }}$ is the rotational viscosity coefficient. The molecular field $H_{i j}$ drives the system towards the equilibrium of $Q_{i j}$ and can be interpreted from the free energy $F$ (alternatively, see Ref. [2]):

$$
H_{i j}=-\frac{\delta F}{\delta Q_{i j}}+\frac{\delta_{i j}}{3} \operatorname{Tr} \frac{\delta F}{\delta Q_{k l}},
$$


where $F$ is written in the Landau-de Gennes form as

$$
\begin{aligned}
F= & \int_{V}\left\{\frac{A}{2} Q_{i j} Q_{j i}+\frac{B}{3} Q_{i j} Q_{j k} Q_{k i}+\frac{C}{4}\left(Q_{i j} Q_{j i}\right)^{2}\right. \\
& \left.+\frac{L}{2} \frac{\partial Q_{i j}}{\partial x_{k}} \frac{\partial Q_{i j}}{\partial x_{k}}\right\} \mathrm{d} V .
\end{aligned}
$$

Derivatives of $Q_{i j}$ in Eq. (A3) describe the effective elastic behavior of the director field, where $L$ is the elastic constant. Summation over the repeated indices is implied. Note that $A, B$, and $C$ are parameters that can be used to tune the nematic phase behavior.

The advection term $S_{i j}$ couples the velocity and nematic ordering:

$$
\begin{aligned}
S_{i j}= & \left(\chi D_{i k}-\Omega_{i k}\right)\left(Q_{k j}+\frac{\delta_{k j}}{3}\right) \\
& +\left(Q_{i k}+\frac{\delta_{i k}}{3}\right)\left(\chi D_{k j}+\Omega_{k j}\right) \\
& -2 \chi\left(Q_{i j}+\frac{\delta_{i j}}{3}\right) Q_{k l} W_{l k},
\end{aligned}
$$

where $D_{i j}$ is the symmetric and $\Omega_{i j}$ the antisymmetric part of the velocity gradient tensor $W_{i j}=\partial_{i} u_{j}$. The alignment parameter $\chi$ depends on the molecular shape and defines the flow-aligning or flow-tumbling regime.

Surface alignment is modeled as follows. For droplets with perpendicular surface alignment (homeotropic anchoring), strong anchoring with fixed radial director at the droplet surface is assumed. Droplets with in-plane degenerate planar alignment (degenerate planar anchoring) are modeled by the surface free energy density:

$$
f_{\mathrm{deg}}=W_{\mathrm{deg}}\left(\tilde{Q}_{i j}-\tilde{Q}_{i j}^{\perp}\right)^{2},
$$

where $\tilde{Q}_{i j}=Q_{i j}+S \delta_{i j} / 2, \tilde{Q}_{i j}^{\perp}=\left(\delta_{i k}-\nu_{i} \nu_{k}\right) \tilde{Q}_{k l}\left(\delta_{k j}-\nu_{k} \nu_{j}\right)$, and $\boldsymbol{\nu}$ is the surface normal. The surface Q-tensor field follows the dynamics

$$
\dot{Q}_{i j}=\Gamma^{\text {surf }}\left(H_{i j}^{\text {surf }}-\frac{\delta_{i j}}{3} \operatorname{Tr} H_{k l}^{\text {surf }}\right),
$$

where

$$
H_{i j}^{\text {surf }}=-\frac{\partial f_{\mathrm{vol}}}{\partial\left(\partial_{k} Q_{i j}\right)} \nu_{k}-\frac{\partial f_{\mathrm{deg}}}{\partial Q_{i j}}
$$

and $f_{\mathrm{vol}}$ is the free energy density expressed in Eq. (A3).

The fluid velocity obeys the incompressibility condition and the Navier-Stokes equation,

$$
\begin{aligned}
\nabla \cdot \boldsymbol{u} & =0, \\
\rho\left(\partial_{t}+u_{k} \partial_{k}\right) u_{i} & =\partial_{j} \Pi_{i j},
\end{aligned}
$$

where $\rho$ is the fluid density and $\Pi_{i j}$ the stress tensor, which consists of a passive and an active part $\Pi_{i j}=\Pi_{i j}^{\text {passive }}+$ $\Pi_{i j}^{\text {active }}$, where

$$
\begin{aligned}
\Pi_{i j}^{\mathrm{passive}}= & -P \delta_{i j}+2 \chi\left(Q_{i j}+\frac{\delta_{i j}}{3}\right) Q_{k l} H_{k l} \\
& -\chi H_{i k}\left(Q_{k j}+\frac{\delta_{k j}}{3}\right)-\chi\left(Q_{i k}+\frac{\delta_{i k}}{3}\right) H_{k j} \\
& -\partial_{i} Q_{k l} \frac{\delta F}{\delta \partial_{j} Q_{k l}}+Q_{i k} H_{k j}-H_{i k} Q_{k j}+2 \eta D_{i j}, \\
\Pi_{i j}^{\mathrm{active}} & =-\zeta Q_{i j} .
\end{aligned}
$$

Here, $P$ is fluid pressure, $\eta$ the isotropic viscosity contribution, and $\zeta$ is the activity parameter characterizing the strength of force dipoles of contractile $(\zeta<0)$ and extensile $(\zeta>0)$ objects.

Coupled equations for the fluid velocity $u_{i}$ and the nematic order $Q_{i j}$ are solved numerically by the hybrid lattice-Boltzmann algorithm [16,28]. The hybrid algorithm consists of an explicit finite-difference method for the Q-tensor evolution [Eq. (A1)], and the D3Q19 lattice Boltzmann model for the Navier-Stokes equation and the compressibility condition [Eqs. (A8) and (A9)]. The nematic stress tensor is implemented in the lattice Boltzmann algorithm as a force contribution with the half-force correction [62]. The spherical droplet cavity is allocated on a rectangular grid, and a no-slip boundary condition is implemented by the bounce-back rule. A radial surface normal is allocated on the surface nodes, and it is used for the calculation of the surface Q-tensor field either for homeotropic or for planar degenerate anchoring. We performed multiple tests to verify that results are not affected by spurious velocities and numerical-methodinduced symmetry or symmetry breaking. For example, in Fig. 1(a), for different random initial conditions, the defect is displaced from the center in different (arbitrary) directions that are typically not along the (rectangular) simulation grid axes. The results of the simulations are expressed in units of elastic constant $L$, nematic correlation length $\xi_{\mathrm{N}}=\sqrt{L /\left(A+B S_{\mathrm{eq}}+\frac{9}{2} C S_{\mathrm{eq}}^{2}\right)}$. where $S_{\mathrm{eq}}$ is the equilibrium nematic degree of order, and nematic intrinsic timescale $\tau_{\mathrm{N}}=\xi_{\mathrm{N}}^{2} / \Gamma^{\text {bulk }} L$. The phase parameters are set to $A=-0.190 L / \xi_{\mathrm{N}}^{2}, B=-2.34 L / \xi_{\mathrm{N}}^{2}$, and $C=1.91 L / \xi_{\mathrm{N}}^{2}$; the nematic is in the aligining regime with $\chi=1$; the isotropic viscosity contribution equals $\eta=1.38 \xi_{\mathrm{N}}^{2} / L \tau_{\mathrm{N}}$; and the strength of the planar degenerate anchoring is $W_{\text {deg }}=6.6 \times 10^{-4} L / \xi_{\mathrm{N}}$, with surface rotational viscosity parameter $\Gamma^{\text {surf }}=0.67 \Gamma^{\text {bulk }} / \xi_{\mathrm{N}}$. The grid resolution is set to $\Delta x=1.5 \xi_{\mathrm{N}}$ and time resolution to $\Delta t=0.057 \tau_{\mathrm{N}}$. The velocity field of the active nematic can be analyzed also by calculating the total angular momentum $\boldsymbol{\Gamma}=\int \rho \mathbf{r} \times \mathbf{u d} V$, where $\mathbf{r}$ is the distance from the droplet center and integration is performed over the whole volume of the active nematic droplet. 
The parameters of the considered 3D active nematic system can be summarized by introducing two selected dimensionless numbers [according to Eqs. (A1) and (A9)]: the Ericksen number $\mathrm{Er}=u R / \Gamma^{\mathrm{bulk}} L$, comparing the viscous terms to the elastic terms, and the activity number $\mathrm{Ac}=\sqrt{\zeta R^{2} / L}$, characterizing the relative strength of activity vs confinement, where $u$ is the maximum velocity in droplets of radius $R$ and activity $\zeta$. In our simulations, we take activities of up to $\zeta \lesssim 0.06 L / \xi_{\mathrm{N}}^{2}$ and droplet radius of $R=136 \xi_{\mathrm{N}}$, which corresponds to $\mathrm{Er} \lesssim 100$ and $\mathrm{Ac} \lesssim 35$.

\section{Tracing and visualization of defects}

Modeling of active nematics gives a continuous threedimensional Q-tensor field and a velocity field on a discrete grid at each time step. To perform the topological analysis, geometry of defect lines is extracted from the Q field. Defects are detected by first finding the point in the sample that has the lowest degree of order $S$. A small nearby sphere with three data points in radius is searched for the lowest $S$, and the line is propagated until it closes the defect loop by meeting the initial point. Visited points are removed from the search space, and the algorithm is repeated until no regions with sufficiently low order are found. This procedure produces polygonal lines that approximate the defect loops, and an associated coordinate frame is constructed, consisting of the tangent $\hat{t}$ and two perpendicular vectors $\hat{t}_{1}$ and $\hat{t}_{2}$. The choice of these two vectors is, in principle, arbitrary, as long as they vary continuously around the loop. For each defect loop segment, the surrounding director field is analyzed on a small circle spanned by the perpendicular vectors $\hat{t}_{1}$ and $\hat{t}_{2}$. Two mutually perpendicular normalized vectors $\hat{n}_{1}$ and $\hat{n}_{2}$ are found, which are used to parametrize the circle on the unit sphere of all directions, visited by the director as traversing the circle. These directions $\hat{n}_{1}$ and $\hat{n}_{2}$ are flipped head to tail if needed so that they vary continuously along the loop. Keeping the signs and orientation of the local coordinate frame consistent with respect to the initial reference point, we overcome the ambiguities inherent in the line field topology. Note that we trace two coordinate frames over the entire loop. The first frame consists of $\hat{t}, \hat{t}_{1}$, and $\hat{t}_{2}$, and the second frame consists of $\hat{n}_{1}, \hat{n}_{2}$, and $\hat{n}_{1} \times \hat{n}_{2}$. The orientations of these frames are represented with quaternions instead of rotational matrices, to retain the full topological information in a closed loop [44]. The $\hat{n}_{1}$ and $\hat{n}_{2}$ pair defines the discs, drawn in Fig. 3(b) to represent the cross section, while the color represents the quantity $\hat{t} \cdot\left(\hat{n}_{1} \times \hat{n}_{2}\right)$ that distinguishes the winding number from the pure $-1 / 2$ profile in red to the pure $+1 / 2$ in blue. A splay-bend parameter is used to visualize the local director around the active defect loops [45].

The velocity field in Fig. 1 is shown by blue arrows above the selected cutoff magnitudes of $v=0.0320 \xi_{\mathrm{N}} / \tau_{\mathrm{N}}$ in (a) at $\zeta=0.003 L / \xi_{\mathrm{N}}^{2}, v=0.127 \xi_{\mathrm{N}} / \tau_{\mathrm{N}}$ in (a) at $\zeta=0.013 L / \xi_{\mathrm{N}}^{2}$, $v=0.195 \xi_{\mathrm{N}} / \tau_{\mathrm{N}}$ in (a) at $\zeta=0.022 L / \xi_{\mathrm{N}}^{2}, v=0.304 \xi_{\mathrm{N}} / \tau_{\mathrm{N}}$ in (b), and $v=0.326 \xi_{\mathrm{N}} / \tau_{\mathrm{N}}$ in (d). The velocity magnitude is additionally represented by a bright green isosurface at similar cutoff values of $v=0.0305 \xi_{\mathrm{N}} / \tau_{\mathrm{N}}$ in (a) at $\zeta=$ $0.003 L / \xi_{\mathrm{N}}^{2}, v=0.125 \xi_{\mathrm{N}} / \tau_{\mathrm{N}}$ in (a) at $\zeta=0.013 L / \xi_{\mathrm{N}}^{2}, v=$ $0.0320 \xi_{\mathrm{N}} / \tau_{\mathrm{N}}$ in (a) at $\zeta=0.192 L / \xi_{\mathrm{N}}^{2}, v=0.297 \xi_{\mathrm{N}} / \tau_{\mathrm{N}}$ in (b), and $v=0.316 \xi_{\mathrm{N}} / \tau_{\mathrm{N}}$ in (d).

[1] M. C. Marchetti, J. F. Joanny, S. Ramaswamy, T. B. Liverpool, J. Prost, M. Rao, and R. A. Simha, Hydrodynamics of Soft Active Matter, Rev. Mod. Phys. 85, 1143 (2013).

[2] A. Doostmohammadi, J. Ignés-Mullol, J. M. Yeomans, and F. Sagués, Active Nematics, Nat. Commun. 9, 3246 (2018).

[3] C. Blanch-Mercader, V. Yashunsky, S. Garcia, G. Duclos, L. Giomi, and P. Silberzan, Turbulent Dynamics of Epithelial Cell Cultures, Phys. Rev. Lett. 120, 208101 (2018).

[4] T. Sanchez, D. T. N. Chen, S. J. DeCamp, M. Heymann, and Z. Dogic, Spontaneous Motion in Hierarchically Assembled Active Matter, Nature (London) 491, 431 (2012).

[5] D. Needleman and Z. Dogic, Active Matter at the Interface between Materials Science and Cell Biology, Nat. Rev. Mater. 2, 17048 (2017).

[6] J. Brugués and D. Needleman, Physical Basis of Spindle Self-Organization, Proc. Natl. Acad. Sci. U.S.A. 111, 18496 (2014).

[7] J. Prost, F. Jülicher, and J.-F. Joanny, Active Gel Physics, Nat. Phys. 11, 111 (2015).

[8] T. B. Saw, A. Doostmohammadi, V. Nier, L. Kocgozlu, S. Thampi, Y. Toyama, P. Marcq, C. T. Lim, J. M. Yeomans, and B. Ladoux, Topological Defects in Epithelia Govern Cell Death and Extrusion, Nature (London) 544, 212 (2017).

[9] K. Kawaguchi, R. Kageyama, and M. Sano, Topological Defects Control Collective Dynamics in Neural Progenitor Cell Cultures, Nature (London) 545, 327 (2017).

[10] G. P. Alexander, B. G. Chen, E. A. Matsumoto, and R. D. Kamien, Colloquium: Disclination Loops, Point Defects, and All that in Nematic Liquid Crystals, Rev. Mod. Phys. 84, 497 (2012).

[11] L. Giomi, M. J. Bowick, X. Ma, and M. C. Marchetti, Defect Annihilation and Proliferation in Active Nematics, Phys. Rev. Lett. 110, 228101 (2013).

[12] S. J. DeCamp, G. S. Redner, A. Baskaran, M. F. Hagan, and Z. Dogic, Orientational Order of Motile Defects in Active Nematics, Nat. Mater. 14, 1110 (2015).

[13] L. Giomi, Geometry and Topology of Turbulence in Active Nematics, Phys. Rev. X 5, 031003 (2015).

[14] J. Urzay, A. Doostmohammadi, and J. M. Yeomans, Multiscale Statistics of Turbulence Motorized by Active Matter, J. Fluid Mech. 822, 762 (2017).

[15] S. Henkes, M. C. Marchetti, and R. Sknepnek, Dynamical Patterns in Nematic Active Matter on a Sphere, Phys. Rev. E 97, 042605 (2018).

[16] A. Doostmohammadi, T. N. Shendruk, K. Thijssen, and J. M. Yeomans, Onset of Meso-Scale Turbulence in Active Nematics, Nat. Commun. 8, 15326 (2017).

[17] A. Maitra, P. Srivastava, M. C. Marchetti, J. S. Lintuvuori, S. Ramaswamy, and M. Lenz, A Nonequilibrium Force Can 
Stabilize 2D Active Nematics, Proc. Natl. Acad. Sci. U.S.A. 115, 6934 (2018).

[18] E. Putzig, G. S. Redner, A. Baskaran, and A. Baskaran, Instabilities, Defects, and Defect Ordering in an Overdamped Active Nematic, Soft Matter 12, 3854 (2016).

[19] K.-T. Wu, J. B. Hishamunda, D. T. N. Chen, S. J. DeCamp, Y.-W. Chang, A. Fernández-Nieves, S. Fraden, and Z. Dogic, Transition from Turbulent to Coherent Flows in Confined Three-Dimensional Active Fluids, Science 355, eaal1979 (2017).

[20] H. Wioland, F. G. Woodhouse, J. Dunkel, and R. E. Goldstein, Ferromagnetic and Antiferromagnetic Order in Bacterial Vortex Lattices, Nat. Phys. 12, 341 (2016).

[21] E. Lushi, H. Wioland, and R. E. Goldstein, Fluid Flows Created by Swimming Bacteria Drive Self-Organization in Confined Suspensions, Proc. Natl. Acad. Sci. U.S.A. 111, 9733 (2014).

[22] M. Theillard, R. Alonso-Matilla, and D. Saintillan, Geometric Control of Active Collective Motion, Soft Matter 13, 363 (2017).

[23] S. P. Thampi, A. Doostmohammadi, T. N. Shendruk, R. Golestanian, and J. M. Yeomans, Active Micromachines: Microfluidics Powered by Mesoscale Turbulence, Sci. Adv. 2, e1501854 (2016).

[24] F. C. Keber, E. Loiseau, T. Sanchez, S. J. DeCamp, L. Giomi, M. J. Bowick, M. C. Marchetti, Z. Dogic, and A. R. Bausch, Topology and Dynamics of Active Nematic Vesicles, Science 345, 1135 (2014).

[25] D. Khoromskaia and G. P. Alexander, Vortex Formation and Dynamics of Defects in Active Nematic Shells, New J. Phys. 19, 103043 (2017).

[26] R. Zhang, Y. Zhou, M. Rahimi, and J. J. de Pablo, Dynamic Structure of Active Nematic Shells, Nat. Commun. 7, 13483 (2016).

[27] P. Guillamat, J. Ignés-Mullol, and F. Sagués, Control of Active Liquid Crystals with a Magnetic Field, Proc. Natl. Acad. Sci. U.S.A. 113, 5498 (2016).

[28] P. Guillamat, Ž. Kos, J. Hardoüin, J. Ignés-Mullol, M. Ravnik, and F. Sagués, Active Nematic Emulsions, Sci. Adv. 4, eaao1470 (2018).

[29] C. C. Maass, C. Krüger, S. Herminghaus, and C. Bahr, Swimming Droplets, Annu. Rev. Condens. Matter Phys. 7, 171 (2016).

[30] T. Gao and Z. Li, Self-Driven Droplet Powered By Active Nematics, Phys. Rev. Lett. 119, 108002 (2017).

[31] E. Tjhung, D. Marenduzzo, and M. E. Cates, Spontaneous Symmetry Breaking in Active Droplets Provides a Generic Route to Motility, Proc. Natl. Acad. Sci. U.S.A. 109, 12381 (2012).

[32] A. Opathalage, M. M. Norton, M. P. N. Juniper, B. Langeslay, S. A. Aghvami, S. Fraden, and Z. Dogic, Self-Organized Dynamics and the Transition to Turbulence of Confined Active Nematics, Proc. Natl. Acad. Sci. U.S.A. 116, 4788 (2019).

[33] S. Zhou, O. Tovkach, D. Golovaty, A. Sokolov, I. S. Aranson, and O. D. Lavrentovich, Dynamic States of Swimming Bacteria in a Nematic Liquid Crystal Cell with Homeotropic Alignment, New J. Phys. 19, 055006 (2017).
[34] D. Nishiguchi, I. S. Aranson, A. Snezhko, and A. Sokolov, Engineering Bacterial Vortex Lattice via Direct Laser Lithography, Nat. Commun. 9, 4486 (2018).

[35] G. Duclos, R. Adkins, D. Banerjee, M. S. E. Peterson, M. Varghese, I. Kolvin, A. Baskaran, R. A. Pelcovits, T. R. Powers, A. Baskaran, F. Toschi, M. F. Hagan, S. J. Streichan, V. Vitelli, D. A. Beller, and Z. Dogic, Topological Structure and Dynamics of Three Dimensional Active Nematics, arXiv:1909.01381.

[36] A. Scholich, S. Syga, H. Morales-Navarrete, F. SegoviaMiranda, H. Nonaka, K. Meyer, W. de Back, L. Brusch, Y. Kalaidzidis, M. Zerial, F. Jülicher, and B. M. Friedrich, Quantification of Nematic Cell Polarity in ThreeDimensional Tissues, arXiv:1904.08886.

[37] C. A. Whitfield, T. C. Adhyapak, A. Tiribocchi, G. P. Alexander, D. Marenduzzo, and S. Ramaswamy, Hydrodynamic Instabilities in Active Cholesteric Liquid Crystals, Eur. Phys. J. E 40, 50 (2017).

[38] T. N. Shendruk, K. Thijssen, J. M. Yeomans, and A. Doostmohammadi, Twist-Induced Crossover from TwoDimensional to Three-Dimensional Turbulence in Active Nematics, Phys. Rev. E 98, 010601 (2018).

[39] R. Hartmann, P. K. Singh, P. Pearce, R. Mok, B. Song, F. Díaz-Pascual, J. Dunkel, and K. Drescher, Emergence of Three-Dimensional Order and Structure in Growing Biofilms, Nat. Phys. 15, 251 (2019).

[40] U. Tkalec, M. Ravnik, S. Čopar, S. Žumer, and I. Muševič, Reconfigurable Knots and Links in Chiral Nematic Colloids, Science 333, 62 (2011).

[41] A. Martinez, M. Ravnik, B. Lucero, R. Visvanathan, S. Žumer, and I. I. Smalyukh, Mutually Tangled Colloidal Knots and Induced Defect Loops in Nematic Fields, Nat. Mater. 13, 258 (2014).

[42] C. Pfleiderer and A. Rosch, Condensed-Matter Physics: Single Skyrmions Spotted, Nature (London) 465, 880 (2010).

[43] D. Foster, C. Kind, P. J. Ackerman, J.-S. B. Tai, M. R. Dennis, and I. I. Smalyukh, Two-Dimensional Skyrmion Bags in Liquid Crystals and Ferromagnets, Nat. Phys. 15, 655 (2019).

[44] S. Čopar, Topology and Geometry of Nematic Braids, Phys. Rep. 538, 1 (2014).

[45] M. Nikkhou, M. Škarabot, S. Čopar, M. Ravnik, S. Žumer, and I. Muševič, Light-Controlled Topological Charge in a Nematic Liquid Crystal, Nat. Phys. 11, 183 (2015).

[46] Y. Hatwalne, S. Ramaswamy, M. Rao, and R. Simha, Rheology of Active-Particle Suspensions, Phys. Rev. Lett. 92, 118101 (2004).

[47] H. H. Wensink, J. Dunkel, S. Heidenreich, K. Drescher, R. E. Goldstein, H. Löven, and J. M. Yeomans, Meso-Scale Turbulence in Living Fluids, Proc. Natl. Acad. Sci. USA 109, 14308 (2012).

[48] B. Martínez-Prat, J. Ignés-Mullol, J. Casademunt, and F. Sagués, Selection Mechanism at the Onset of Active Turbulence, Nat. Phys. 15, 362 (2019).

[49] P. W. Ellis, D. J. G. Pearce, Y.-W. Chang, G. Goldsztein, L. Giomi, and A. Fernandez-Nieves, Curvature-Induced Defect Unbinding and Dynamics in Active Nematic Toroids, Nat. Phys. 14, 85 (2018). 
[50] See Supplemental Material at http://link.aps.org/ supplemental/10.1103/PhysRevX.9.031051 for Supplemental Figures and Videos.

[51] X. Wang, Y.-K. Kim, E. Bukusoglu, B. Zhang, D. S. Miller, and N. L. Abbott, Experimental Insights into the Nanostructure of the Cores of Topological Defects in Liquid Crystals, Phys. Rev. Lett. 116, 147801 (2016).

[52] G. Posnjak, S. Čopar, and I. Muševič, Hidden Topological Constellations and Polyvalent Charges in Chiral Nematic Droplets, Nat. Commun. 8, 14594 (2017).

[53] R. Voituriez, J. F. Joanny, and J. Prost, Spontaneous Flow Transition in Active Polar Gels, Europhys. Lett. 70, 404 (2005).

[54] R. Green, J. Toner, and V. Vitelli, Geometry of Thresholdless Active Flow in Nematic Microfluidics, Phys. Rev. Fluids 2, 104201 (2017).

[55] K. Kruse, J. F. Joanny, F. Jülicher, J. Prost, and K. Sekimoto, Asters, Vortices, and Rotating Spirals in Active Gels of Polar Filaments, Phys. Rev. Lett. 92, 078101 (2004).
[56] J. Milnor, Morse Theory (Princeton University Press, Princeton, New Jersey, 1963).

[57] M. B. Hindmarsh and T. W. B. Kibble, Cosmic Strings, Rep. Prog. Phys. 58, 477 (1995).

[58] T. Ishikawa and O. D. Lavrentovich, Crossing of Disclinations in Nematic Slabs, Europhys. Lett. 41, 171 (1998).

[59] B. G. Chen, P. J. Ackerman, G. P. Alexander, R. D. Kamien, and I. I. Smalyukh, Generating the Hopf Fibration Experimentally in Nematic Liquid Crystals, Phys. Rev. Lett. 110, 237801 (2013).

[60] L. Metselaar, A. Doostmohammadi, and J. M. Yeomans, Topological States in Chiral Active Matter: Dynamic Blue Phases and Active Half-Skyrmions, J. Chem. Phys. 150, 064909 (2019).

[61] A. N. Beris and B. J. Edwards, Thermodynamics of Flowing Systems with Internal Microstructure (Oxford University Press, New York, 1994).

[62] T. Krüger, H. Kusumaatmaja, A. Kuzmin, O. Shardt, G. Silva, and E. M. Viggen, The Lattice Boltzmann Method (Springer International Publishing, Switzerland, 2017). 